$$
\begin{aligned}
& \text { Pontifícia Universidade Católica } \\
& \text { DO RIO DE JANEIRO }
\end{aligned}
$$

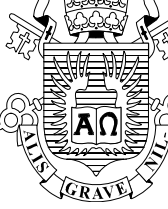

Maristela Provedel de Carvalho

O mundo que eu invento, o mundo que me inventa: vestígios de um self perdido

\title{
Dissertação de Mestrado
}

\begin{abstract}
Dissertação apresentada como requisito parcial para obtenção do grau de Mestre pelo Programa de Pósgraduação em Psicologia do Departamento de Psicologia da PUC-Rio.
\end{abstract}

Orientadora: Profa. Maria Inês Garcia de Freitas Bittencourt

Rio de Janeiro 


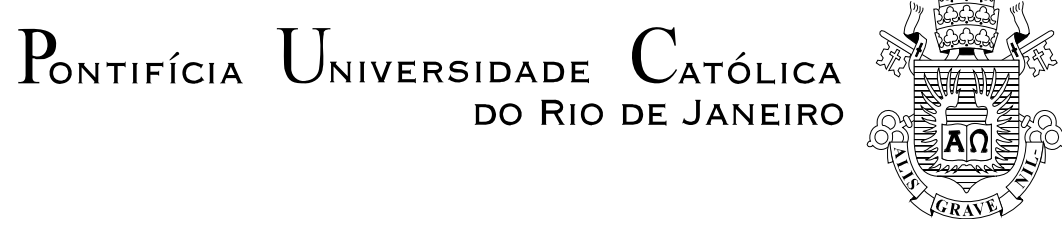

Maristela Provedel de Carvalho

O mundo que eu invento, o mundo que
me inventa: vestígios de um self perdido

Dissertação apresentada como requisito parcial para obtenção do grau de Mestre pelo Programa de Pós-Graduação em Psicologia Clínica do Departamento de Psicologia do Centro de Teologia e Ciências Humanas da PUC-Rio. Aprovada pela Comissão Examinadora abaixo assinada.

Prof $^{\text {a }}$ Maria Inês Garcia de Freitas Bittencourt Orientadora

Departamento de Psicologia - PUC-Rio

Prof ${ }^{a}$. Flavia Sollero de Campos

Departamento de Psicologia - PUC-Rio

Prof. Alexandre Abranches Jordão IBMR-RIO

Prof. Paulo Fernando Carneiro de Andrade Coordenador Setorial de Pós-Graduação

e Pesquisa do Centro de Teologia e Ciências Humanas - PUC-Rio

Rio de Janeiro, I 2010. 
Todos os direitos reservados. É proibida a reprodução total ou parcial do trabalho sem autorização da autora, da orientadora e da universidade.

\section{Maristela Provedel de Carvalho}

Graduou-se em Psicologia Clínica e Bacharelado pela PUC-Rio em 2000. Completou a Formação Psicanalítica na SPCRJ (Sociedade de Psicanálise da Cidade do Rio de Janeiro) em 2005, sendo atualmente membro efetivo desta. Trabalha em seu consultório particular desde o ano de 2000 atendendo crianças, jovens, adultos e adultos de maior idade.

Ficha Catalográfica

Carvalho, Maristela Provedel de

O mundo que eu invento, o mundo que me inventa: vestígios de um self perdido / Maristela Provedel de Carvalho; orientadora: Maria Inês Garcia de Freitas Bittencourt. - 2009.

112 f. ; $30 \mathrm{~cm}$

Dissertação (Mestrado em Psicologia)-Pontifícia Universidade Católica do Rio de Janeiro, Rio de Janeiro, 2009.

Inclui bibliografia

1. Psicologia - Teses. 2. Autenticidade. 3. Ética. 4. Estética. 5. Self. 6. Astúcia. 7. Bricolagem. I. Bittencourt, Maria Inês Garcia de Freitas. II. Pontifícia Universidade Católica do Rio de Janeiro. Departamento de Psicologia. III. Títıln 
Para Maria Uliana Provedel, que de Venda Nova do Imigrante, me mostrou o mundo. 


\section{Agradecimentos}

A minha orientadora Professora Maria Inês Garcia de Freitas Bittencourt pelo estímulo para a realização deste trabalho.

À PUC-Rio pela Bolsa de Isenção (VRAc).

A Flavia Sollero e ao Alexandre Jordão pelo trabalho de participarem desta jornada.

Ao Cid Merlino, pelo auxílio luxuoso de Freud em alemão.

A Luiza Provedel Martins Moreira, pelas precisas e elegantes traduções e revisão do texto.

A Maria Provedel Martins Moreira pelo carinho e pela paciência.

Ao Edu Simões pelo companheirismo e pela força nas horas difíceis. 


\section{Resumo}

Carvalho, Maristela Provedel de; Bittencourt, Maria Inês Garcia de Freitas (Orientadora). 0 mundo que eu invento, o mundo que me inventa: vestígios de um self perdido. Rio de Janeiro, 2009. 112p. Dissertação de Mestrado - Departamento de Psicologia, Pontifícia Universidade Católica do Rio de Janeiro.

Este trabalho pretende analisar, a partir de questões surgidas na prática clínica, a relação entre dois mundos: o mundo particular do indivíduo e o mundo externo a esse indivíduo, representado pelas chamadas sociedades pós-modernas. A idéia de um self perdido é para nós uma imagem representativa do que se passa na contemporaneidade quando as pessoas se esforçam para conseguir ser uma unidade nas gigantescas comunidades. Através de vinhetas clínicas ilustrativas vamos entrelaçar as narrativas pessoais com pontuações advindas da psicanálise e das ciências sociais. Procura-se observar como as sociedades tratam as pessoas e o quanto de senso crítico pode haver por parte destas acerca do mundo em que vivem. Entre tantas possibilidades de ser, uma em especial chama a nossa atenção pela forma astuciosa de pensar e agir. Abordaremos aqui um perfil de pessoas que conseguem transitar pelos excessos que as megalópoles despejam sobre elas utilizando para isso um filtro especial que lhes permite preservar o que possuem de mais precioso: sua autenticidade.

\section{Palavras-chave}

Autenticidade; ética; estética; self; astúcia; bricolagem. 


\section{Abstract}

Carvalho, Maristela Provedel de; Bittencourt, Maria Inês Garcia de Freitas (Advisor). The world $I$ invent, the world that invents me: remains of a lost self. Rio de Janeiro, 2009. 112p. MSc Dissertation - Departamento de Psicologia, Pontifícia Universidade Católica do Rio de Janeiro.

This dissertation aims to analyze, in view of matters observed in the clinic, the relationship between two worlds: the world particular to the individual and the world external to that individual, represented by the so-called postmodern societies. The idea of a lost self is for us an image that represents what has been happening nowadays when people attempt to be a unity in our enormous communities. Using illustrative examples from the clinic, we will intertwine contemporary people's narratives with observations from Psychoanalysis and Social Sciences. We want to observe how societies treat their inhabitants and how much critical thinking can people have concerning the world in which they live. Among so many possibilities of being there is one in special that calls our attention because of its cunningness in thinking and acting. We will approach here this profile of people who manage to journey through the excesses the megalopolis pour down on them using a special filter that allows them to preserve what they have of most precious: their authenticity.

\section{Keywords}

Authenticity; ethics; esthetics; self; cunningness; bricolage. 


\section{Sumário}

$\begin{array}{ll}\text { 1. Introdução } & 10\end{array}$

2. O indivíduo na sociedade dos excessos $\quad 17$

2.1. As grandes cidades e a cultura do consumo 26

2.2. Cinco sentidos e um sujeito 33

2.2.1. O paladar $\quad 36$

2.2.2. O olfato 37

2.2.3. A visão 38

2.2.4. O tato 39

2.2.5. A audição 39

2.3. Um abrigo para o ser 40

2.3.1. Experiências estéticas e memórias 44

2.3.2. Ser uma unidade em uma comunidade 53

3. Pequenos atalhos para um self perdido $\quad 58$

3.1. Vida subjetiva e a relação com o sentido de realidade $\quad 60$

3.2. A busca por uma permanência: o autêntico e o artificial 64

3.3. Composições de subjetividade para os tempos atuais 68

$\begin{array}{ll}\text { 3.3.1. Myrtille } & 71\end{array}$

3.3.2. Esther $\quad 72$

3.3.3. Violet Rose $\quad 73$

3.3.4. Comentários $\quad 75$

3.4. A elasticidade do eu $\quad 81$

4. Considerações finais $\quad 87$

5. Referências bibliográficas 107

$\begin{array}{ll}\text { Anexos } & 110\end{array}$ 
En la escalera de la Torre de la Vitoria, habita desde el principio del tiempo el A Bao A Qu, sensible a los valores de las almas humanas. Vive en estado letárgico, en el primer escalón, y sólo goza de vida consciente cuando alguien sube la escalera. La vibración de la persona que se acerca le infunde vida, y una luz interior se insinúa en él. (...) Testimonio de su sensibilidad es el hecho de que sólo logra su forma perfecta en el último escalón, cuando el que sube es un ser evolucionado espiritualmente. (...) El A Bao A Qu sufre cuando no puede formarse totalmente y su queja es un rumor apenas perceptible, semejante al roce de la seda. (...).

El libro de los seres imaginarios, Jorge Luis Borges 